\title{
Structural and Molecular Compartmentation in the Cerebellum
}

\author{
Richard Hawkes, Steven Blyth, Vijay Chockkan, David Tano, Zhongqi Ji and Christa Mascher
}

\begin{abstract}
Most descriptions treat the cerebellum as a uniform structure, and the possibility of important regional heterogeneities in either chemistry or physiology is rarely considered. However, it is now clear that such an assumption is inappropriate. Instead, there is substantial evidence that the cerebellum is composed of hundreds of distinct modules, each with a precise pattern of inputs and outputs, and expressing a range of molecular signatures. By screening a monoclonal antibody library against cerebellar polypeptides we have identified antigens - zebrins - that reveal some of the cerebellum's covert heterogeneity. This article reviews some of these findings, relates them to the patterns of afferent connectivity, and considers some possible mechanisms through which the modular organization may arise.
\end{abstract}

RÉSUMÉ: Compartimentation structurale et moléculaire du cervelet. La plupart des descriptions représentent le cervelet comme une structure uniforme et l'on considère rarement qu'il puisse exister une hétérogénéité chimique ou physiologique régionale importante. Cependant, il est maintenant évident qu'une telle supposition est inappropriée. Il existe des données importantes montrant que le cervelet est composé de centaines de modules distincts, chacun ayant un patron précis d'entrée et de sortie et exprimant un éventail de signatures moléculaires. À partir d'une librairie d'anticorps monoclonaux dirigés contre des polypeptides cérébelleux, nous avons identifié des antigènes - les zébrines - qui révèlent une partie de l'hétérogénéité cachée du cervelet. Dans cet article, nous revoyons quelques unes de ces observations en établissant leurs relations avec les patrons de connectivité afférente et nous discutons des mécanismes pouvant donner naissance à cette organisation modulaire.

Can. J. Neurol. Sci. 1993; 20 (Suppl. 3): S29-S35

When the cerebellar cortex is analyzed by using conventional techniques clear cytoarchitectonic boundaries are not seen. This has led to models of cerebellar organization and function that emphasize the extreme apparent uniformity of structure. However, receptive field mapping gives a completely different picture, in which the homogeneity of cortical structure fragments into an elaborate mosaic of functional patches. ${ }^{1,2}$ How are these contradictory views to be reconciled? We have assumed that the cytology is misleading and that the apparently uniform cerebellar cytoarchitecture actually conceals significant molecular complexity. To find this putative complexity we constructed a monoclonal antibody (mab) library against molecules in the adult and developing cerebellum, and screened for antigens that are expressed non-uniformly. 3,4

We have identified several antigens that are non-uniformly distributed in the adult rodent cerebellum - the zebrins - and in this review we will concentrate upon just two, zebrin I and zebrin II. On Western blots of cerebellar homogenates mab anti-zebrin I recognizes a $120 \mathrm{kD}$ polypeptide, ${ }^{3}$ and mab antizebrin II recognizes a $36 \mathrm{kD}$ polypeptide..$^{5}$ In neither case has the function of the antigen been securely identified. Both zebrin I and zebrin II are Purkinje cell specific in the cerebellum, although weak zebrin I immunoreactivity is found in a sub-class of astrocytes elsewhere in the brain (e.g., ${ }^{6}$ ).
Zebrins are remarkable in that they are expressed selectively by a subset of Purkinje cells. The zebrin $1 / \mathrm{II}^{+}$sub-population is arranged into parasagittal bands that extend throughout the vermis and hemispheres, interdigitated by similar bands of zebrin I/II- Purkinje cells. Zebrin I and zebrin II are always coexpressed. Detailed analyses of zebrin compartmentation in several species reveals one zebrin $\mathrm{I}_{\mathrm{II}} \mathrm{I}^{+}$band abutting the midline at each side $\left(\mathrm{Pl}^{+}\right)$, and either 6 or 7 other bands running laterally, two in each hemivermis $\left(\mathrm{P}^{+}, \mathrm{P}^{+}\right)$one in each paravermian region $\left(\mathrm{P}^{+}\right)$and 3 or 4 others in the hemispheres (P5 - $\mathrm{P} 7^{+}$ $\left.\left(\mathrm{P}^{+}\right)\right)$. The zebrin $\mathrm{I} / \mathrm{II}^{-}$compartments are named according to the positive compartment immediately medial (rat: 3, 4, 5; opossum - 7; mouse - Eisenman and Hawkes, in preparation). An example of zebrin-stained section through the mouse cerebellum is shown in Figure 1, and a reconstruction of the cerebellum is illustrated in Figure 2.

Molecular heterogeneity in the cerebellar cortex is highly conserved across phyla. Anti-zebrin II immunoreactivity reveals similar parasagittal compartments in the rat, mouse, opossum, cat and chick. Furthermore, zebrin II is expressed selectively by Purkinje cell subsets in a variety of teleost fishes and Western blotting shows an apparently identical antigen in both fish and rat. ${ }^{5.8}$ In human cerebellum, both zebrin ${ }^{+}$and zebrin- Purkinje cells are present but their arrangement has not been worked out. ${ }^{9}$

From the Department of Anatomy and Neuroscience Research Group, University of Calgary, Calgary

Reprint requests to: Dr. R.B. Hawkes, Department of Anatomy, Faculty of Medicine, University of Calgary, 3330 Hospital Drive N.W., Calgary, Alberta, Canada T2N 4N 1 

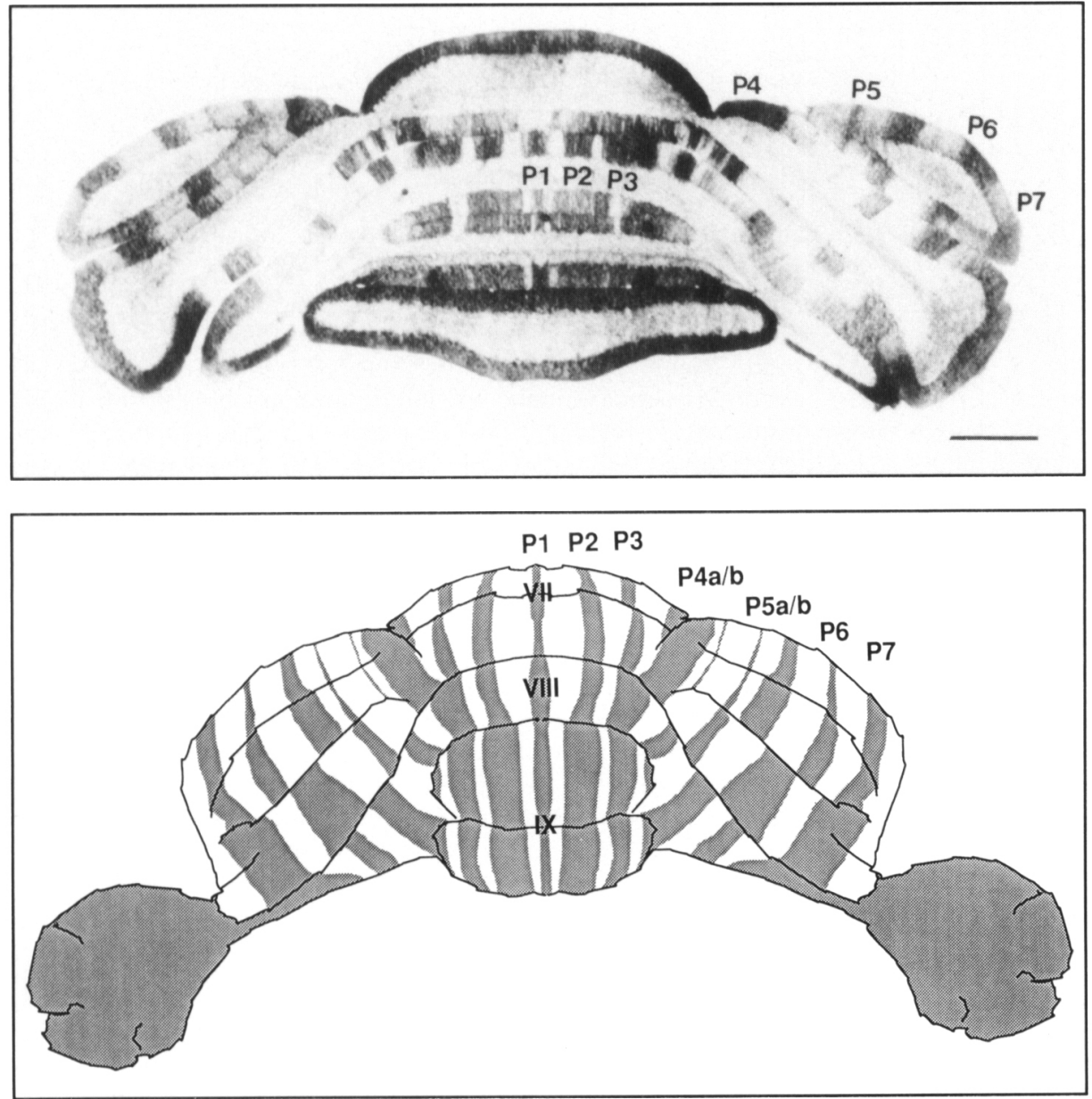

Figure 1 - A frontal section through the adult mouse cerebellar cortex immunoperoxidase stained by using mab anti-zebrin II. The alternating bands of zebrin-positive and zebrin-negative Purkinje cells are clearly seen (the positive bands are labelled $P$ I $-P 7$ ). Scale bar $=2 \mathrm{~mm}$.
Besides zebrins, several other molecular markers have a nonuniform distribution in the adult mammalian cerebellum. Their patterns of compartmentation are similar: these include the enzymes $5^{\prime}$-nucleotidase, ${ }^{10}$ acetylcholinesterase, 11 and cytochrome oxidase.$^{12}$

These data suggest that there are just two classes of Purkinje cell, as defined by their molecular signatures. However, there is good reason to think that this is an underestimate. On the one hand, several markers have been described in the cerebellum that do not fit the parasagittal compartmentation map of the zebrins (e.g., antigen B : 13). On the other hand, there are a few markers that seem to follow the zebrin-defined compartment boundaries but reveal unexpected complexity. One such is the acetylated ganglioside recognized by monoclonal antibody $\mathrm{P}$ Path, ${ }^{14}$ which is primarily a marker of Purkinje cells in the zebrin-negative compartments, and as such, is the first such "positive" marker of the P- set. However, P-path immunoreactivity is also co-expressed by a subset of the zebrin ${ }^{+}$Purkinje cells, for example those of the $\mathrm{P}^{+}$band. This further sub-division of the zebrin ${ }^{+}$compartments may be the first example of a more profound heterogeneity.

\section{Correspondence Between Purkinje Cells and Afferents}

For the zebrin I/II distribution to be central to cerebellar organization, it must bear a close relationship to the patterns of afferent and efferent connectivity. To explore this issue, zebrin immunocytochemistry was combined with anterograde tracer techniques to compare afferent terminal field distributions to the pattern of Purkinje cell compartmentation.

\section{Climbing Fibers: the Olivocerebellar Projection}

Adjacent cell clusters in the inferior olivary complex do not necessarily project to adjacent regions of the cerebellar cortex, (e.g., projections to the uvula:15). As a result, small injections of anterograde tracer into discrete regions of the inferior olivary complex label parasagittal bands of climbing fibers in the cerebellar cortex. It is imperative to determine how the intrinsic chemistry of the cerebellar cortex relates to these innervation patterns.

The climbing fiber compartments and the zebrin I bands seem to respect common boundaries. Both are in reproducible parasagittal bands, and their boundaries are aligned. ${ }^{16}$

\section{Mossy Fibers: the Spinocerebellar Projection}

The second major afferent projections to the cerebellum are the mossy fibers. Unlike the olivocerebellar projection, where there is a direct synaptic interaction and precise compartment alignment, the mossy fiber-Purkinje cell interaction is indirect, and may therefore be architecturally less-precise. The crucial question is: do the mossy fiber terminal fields respect the Purkinje cell compartments? We have compared the terminal field distributions of mossy fibers originating at different spinal levels to the Purkinje cell compartmentation (e.g., 17; Ji and Hawkes, MS in preparation). Examples from the anterior lobe vermis are illustrated in Figure 3. There are differences in mossy 
fiber terminal field position depending upon the site of origin of the axons. For example, in the vermis of lobule III the terminal distribution becomes more restricted as we proceed from rostral to caudal. Thus, anterograde tracer injections in cervical regions show little restriction of the terminal field into patches, thoracic injections reveal broad symmetrical bands, and lumbar injections show similar, but narrower bands. For mossy fiber terminal field patches both of thoracic and lumbar origin, there is a close alignment with the Purkinje cell bands. For example, both strictly stop at the lateral edge of $\mathrm{P}^{+}$while they extend to varying degrees medially into $\mathrm{PI}$-.

The identification of parasagittal compartments of Purkinje cells tightly coupled to climbing fiber bands, serves in part to explain how the ostensibly uniform cerebellar cortex might encode complex somatotopic maps. The further sagittal subdivision of the zebrin compartments by the mossy fiber terminal fields makes the anatomical compartment widths compatible with the physiological compartments revealed by using receptive field mapping.

There are three ways that these longitudinal bands may be subdivided mediolaterally to give the patchy mosaic of receptive fields (Figure 4):

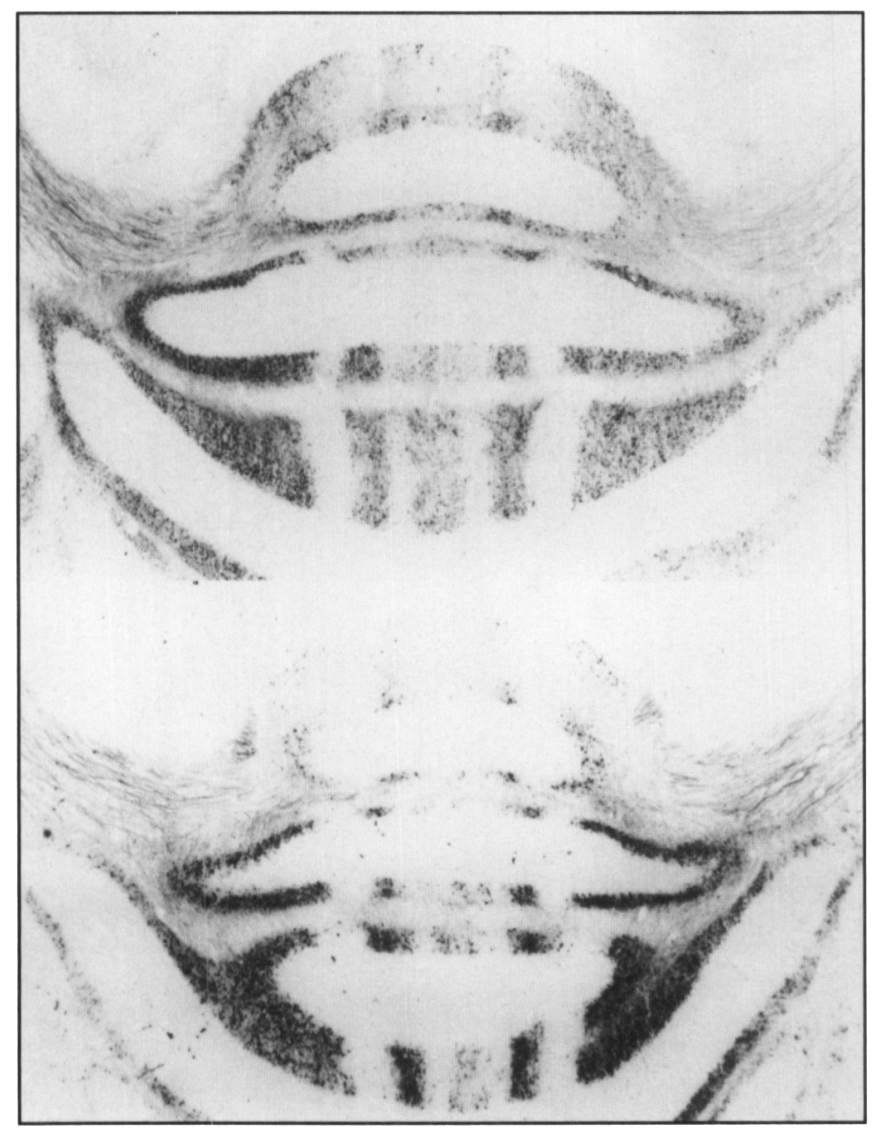

Figure 3-Two mossy fiber terminal field distributions in the anterior lobe of the adult rat, as revealed using the anterograde transport of wheat germ agglutinin conjugated to horseradish perovidase. Injections were placed into either the thoracic (upper panel), or the lumbar (lower panel) regions of the spinal cord. The animals were sacrificed two days later and processed for anterograde transport: for details of the method, see Ref. ${ }^{17}$. Scale bar $=400 \mu \mathrm{m}$. i) The natural cerebellar foliation, which provides a natural means by which afferent inputs can be directed to one or other cortical territory e.g., the restriction of spinocerebellar terminals to a few spinal receiving areas (Figure 4a).

ii) Some mossy fiber terminal fields apparently shift position as they progress from the dorsal to ventral surface of a lobule, or are only present on one face. For example, in the dorsal surface of lobule II the thoracic terminal fields are centered more-or-less beneath the $\mathrm{P}^{+}$compartments. In contrast, on the ventral face they lie in $\mathrm{P}-$ compartments. ${ }^{16}$ As a result, the afferent innervation of the same compartment can be different between dorsal and ventral faces of the lobule (Figure 4b).

iii) Some mossy fibers penetrate less deeply than others into the lobule, and therefore mossy fibers from different sources are stacked along the axis of the folium, leading inevitably to the patchy segregation of afferent information (Figure $4 \mathrm{c}$ ).

Thus, the natural separation of afferents that occurs between lobules at the depths of the sulci and the mossy fiber distribution within an individual lobule together may subdivide Purkinje cell compartments into patches with different patterns of afferent innervation.

Although the cerebellar cortex appears to be subdivided into multiple patches, these may in turn be regrouped into sets by several mechanisms:

- both mossy fiber and climbing fiber collateral branches extend rostrocaudally between lobules, thereby providing common afferent input to widely distant modules. These projections tend to be aligned sagittally, suggesting that most such branching occurs within the same Purkinje cell compartment.

- the parallel fibers extend laterally over several modules

- the Purkinje cell recurrent axon collaterals, although confined primarily to within one Purkinje cell band, do extend into adjacent bands as well

- some mossy fibers branch to send collaterals laterally. When the carbocyanine dye Dil is used as a tracer in the cerebellar cortex, the pattern of dye diffusion reveals discrete patches of dye labelling in the granular layer distant from the injection site (e.g., Figure 5). Seen at high magnification, the labelled structures resemble mossy fiber terminals. The most straightforward way to account for these is to postulate a branching mossy fiber going to both the injection site and the secondary patch.

\section{DEVElopMent of Compartmentation}

\section{Perinatal Markers}

A selection of immunological markers is now available to monitor the maturation of Purkinje cells in rodents. In rat, the earliest antigen to be expressed is cyclic guanosine $3^{\prime}: 5^{\prime}$ phosphate-dependent protein kinase (cPK) that appears for the first time at embryo day 17 in a subset of Purkinje cells ${ }^{19}$ (the Purkinje cell terminal mitoses are between embryo days 12 and 15). Differential expression by a subset of Purkinje cells is continued until birth. In the first five days postnatally, antigen expression gradually extends to include all Purkinje cells, and differential expression is not seen in the adult. In the opossum Monodelphis, the protracted postnatal development may allow a direct comparison of early and late compartmentation antigens. An example of early compartmentation in the opossum 


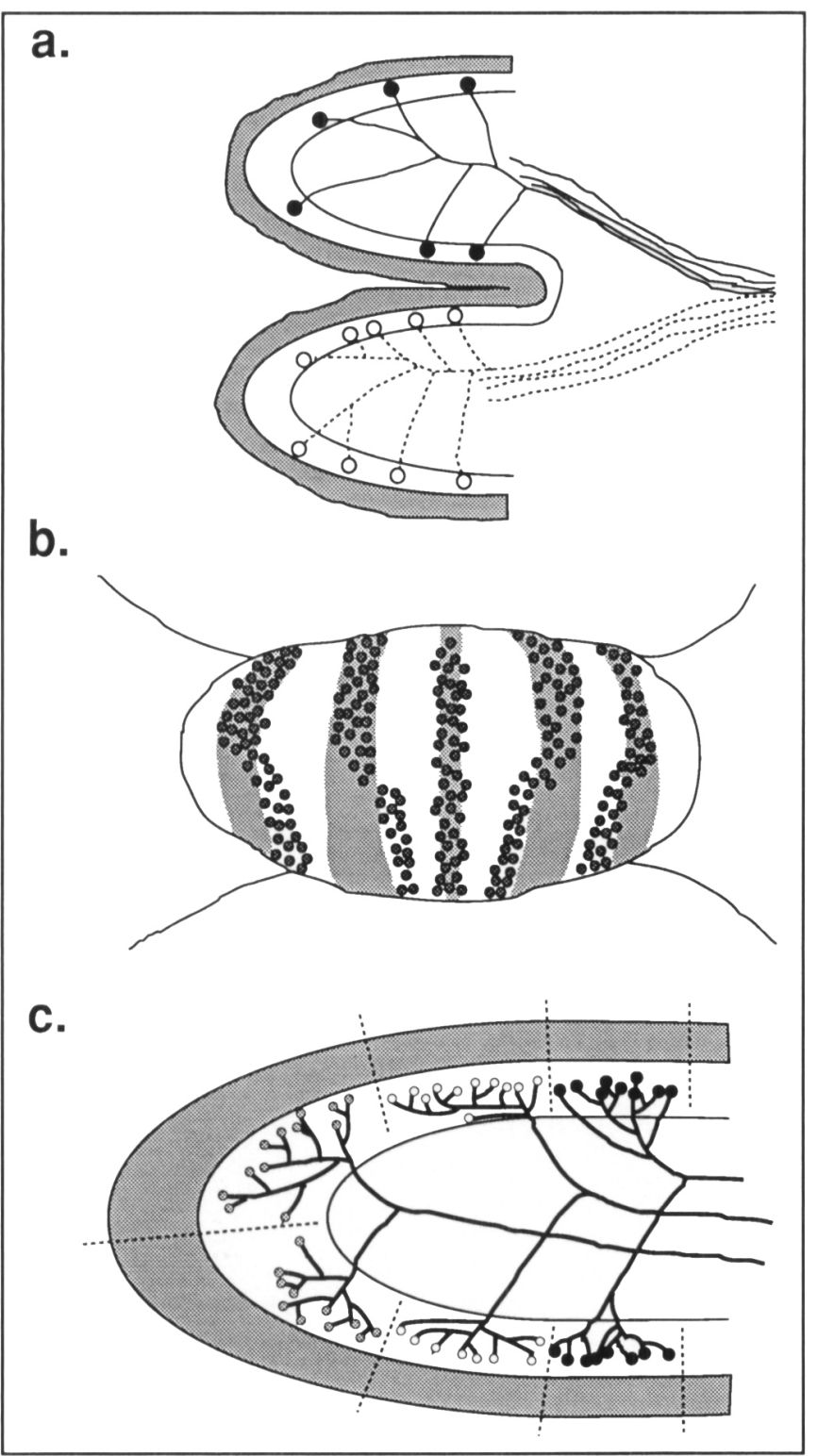

Figure 4 - Three cartoons to illustrate ways by which longitudinal Purkinje cell compartments may be subdivided into modules: A) A sagittal view of two lobules that receive different mossy fiber inputs. The differential branching of ingrowing axons between lobules can result in the segregation of different mossy fiber receiving areas. B) A frontal view of a single lobule: the zebrin ${ }^{+}$compartments are shaded, and mossy fiber terminals indicated by filled circles. In several cases, the mossy fiber terminal fields have been seen to shift with respect to the zebrin compartments as they progress around a lobule. Such shifts subdivide zebrin compartments mediolaterally into receiving and non-receiving patches. $C$ ) If mossy fiber axons grow sequentially into a cerebellar lobule, one result will be the pattern of innervation illustrated here in a sagittal view, with axon stacking within the lobule resulting in symmetrical patches of inputs on the dorsal and ventral faces. If the successive ingrowing axons carry different information, this will naturally generate a patchy functional organization. Our preliminary Dil labelling experiments provide evidence for this sort of organization: dye injections onto the dorsal surface of lobules often generates a discrete corresponding patch of dye labelling on the ventral face (Blyth and Hawkes, unpublished observations). cerebellum as revealed by using anti-calbindin is illustrated in (Figure 6) (Chockkan, Jacobson, and Hawkes: MS in preparation). Studies are in progress to compare calbindin and zebrin II compartmentation patterns.

Several other perinatal compartment antigens have also been described. Anti-vitamin D-dependent calcium binding protein (CaBP) antiserum and anti-Purkinje cell specific glycoprotein (PSG) both selectively recognize transient perinatal Purkinje cell populations. ${ }^{20}$ As for $\mathrm{cPK}$ expression, the heterogeneity seems to reflect timing differences in the onset of expression, and disappears during the first few postnatal days.

\section{Zebrin I/II:}

Both zebrin I and zebrin II follow an identical developmental timetable in the rat. We have identified three stages in the expression of the zebrin I epitope, an initial phase in which all Purkinje cells are zebrin $\mathrm{I}^{-}$, an intermediate stage in which all become zebrin $\mathrm{I}^{+}$, and the final, adult stage of selective expression by parasagittal bands of cells. ${ }^{21}$ The late expression of zebrin I during corticogenesis precludes it from playing a direct

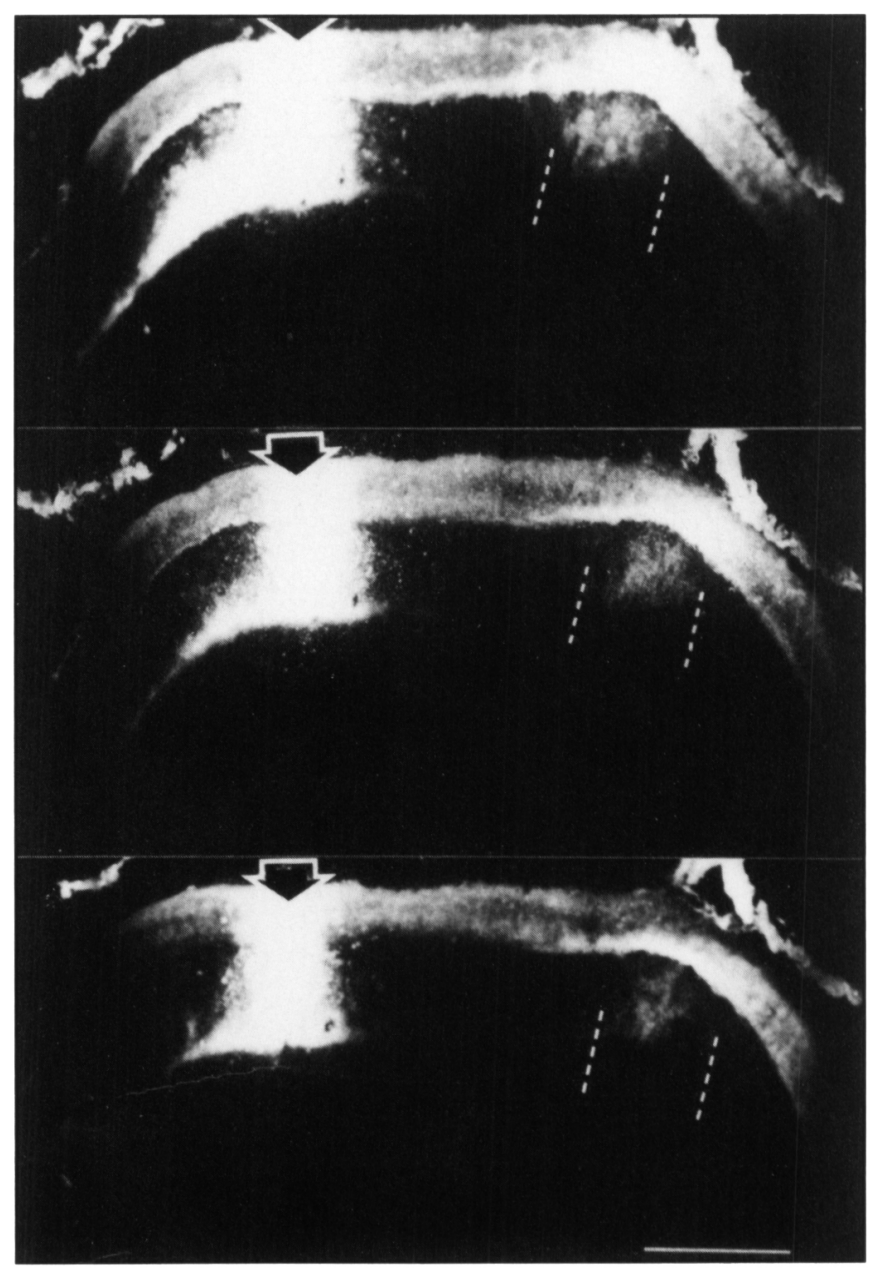

Figure 5 - A small crystal of the carbocyanine dye Dil was placed into the molecular layer of the cerebellar cortex of an aldehyde. fixed adult rat. After one week, the tissue was sectioned. Three serial frontal sections (each $50 \mu \mathrm{m}$ thick and $100 \mu \mathrm{m}$ apart) reveal an apparent connection between the injection site (arrowhead) and a patch of mossy fiber terminals located contralaterally (indicated by dotted lines). Scale bar $=I \mathrm{~mm}$. 


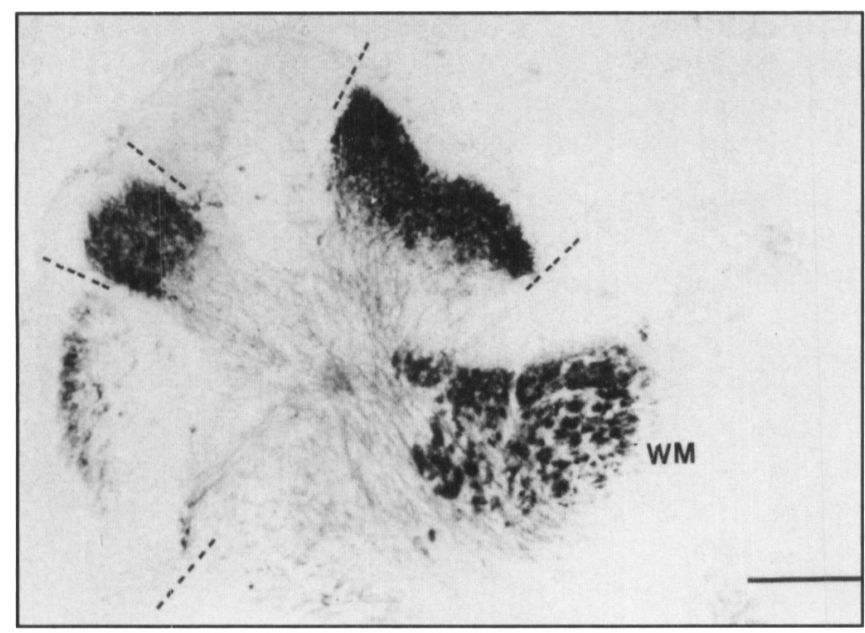

Figure 6-A frontal section through the cerebellar hemisphere of the Brazilian opossum Monodelphis at postnatal day 15 (equivalent roughly to one week postnatal in the rat-see Ref. ${ }^{8}$ ), immunoperoxidase stained with anti-calbindin. Calbindin immunoreactivity in the cerebellum is Purkinje cell-specific, and reveals several clearlydelineated cell clusters (indicated by dotted lines). The immunoreactive Purkinje cell axons can be followed out to the main white matter bundle $(W M)$. Scale bar $=200 \mu \mathrm{m}$.

role in the initial encoding of positional information since some of the parasagittal zonation of the cerebellar cortex is already established at birth (e.g., ${ }^{22.23}$ ).

Zebrin $\mathrm{I} / \mathrm{II}$ induction begins at the median posterior vermis at P6. By P7, clusters of immunoreactive Purkinje cells are clearly evident in the posterior lobe vermis, but the anterior lobe vermis and the hemispheres are unreactive. Expression spreads in a discontinuous, saltatory fashion, with a clear boundary - on the ventral face of lobule VIII - dividing the vermis at P7 into zebrin $\mathrm{I}^{+}$and zebrin $\mathrm{I}^{-}$territory. In a second stage, zebrin expression appears both anteriorly and laterally so that, about $5-7$ days after the onset, all Purkinje cells are zebrin $\mathrm{I}^{+}$. The transient banding pattern has disappeared by $\mathrm{P} 12$, but differences in staining intensity indicate the positions of the future $\mathrm{P}^{+} / \mathrm{P}^{-}$compartments. The relationship between the early and mature compartmentation is unclear. The factors that control the induction and spread of zebrin I expression in the Purkinje cell population are unknown.

In the opossum, the expression of zebrin II during development closely resembles that in the rat. Monodelphis is born after only 14 days in utero, and remains in the maternal pouch for several weeks thereafter. When rat and opossum zebrin II expression timetable are compared, the correspondence is remarkable (Figure 5): the first immunoreactivity appears at P20 (equivalent to rat P6), uniform expression by all Purkinje cells is seen at P28, and zebrin $\mathrm{II}^{+} /$bands become obvious at about $P 35$. The final maturation of the band array appears to be more protracted than in rat, and the adult appearance is only achieved after P56. ${ }^{7}$ The transient expression of zebrin II in some Purkinje cells destined to be zebrin $\mathrm{II}^{-}$in adults is also phylogenetically conserved in fish. This implies that this developmental program is not a vagary of the mammalian cerebellum but is instead a fundamental feature of Purkinje cell maturation. Since independent antigens have identical patterns of expression, there must be major changes in gene expression in the Purkinje cell subsets late in their development. Studies underway of the regulation of zebrin $\mathrm{Il}$ expression at the genomic level should help to clarify this issue.

In some fish, for example the weakly-electric teleost Eigenmannia - a further level of complexity is found, with zebrin $\mathrm{Il}^{-}$regions differing in their modes of expression, in that some Purkinje cells are never zebrin $\mathrm{II}^{+} .8 .24$ This is the first time that a complete lack of zebrin II expression throughout Purkinje cell development has been observed, and indicates that zebrin II expression is not obligatory in all vertebrates.

There remain obvious gaps in our knowledge concerning the timetable of Purkinje cell biochemical compartmentation in mammals. Three stages have been identified: perinatal (e.g., cPK immunoreactivity: $\left.{ }^{19.20}\right)$, the early zebrin $\mathrm{I}^{+} /$zebrin $\mathrm{I}^{-}$clusters in the posterior lobe vermis (P6 - P9) and the mature display of bands and patches (from P15). How do these three relate to one another? Unfortunately, the three patterns are never coexistent. The early clusters revealed by using anti-zebrin $I$ in rats and mice do not appear until the cPK is distributed uniformly, and then they are swamped by the phase of global zebrin I expression at around P12. Previous studies of chemical compartmentation during perinatal development concluded that multiple overlapping families of Purkinje cells were present, and all markers do not respect common boundaries. ${ }^{19.20}$ However, our preliminary studies of calbindin and zebrin co-expression in neonatal opossums does suggest a set of common boundaries, so substantial variation in the lineage restrictions of different markers may be involved.

It seems likely that cerebellar compartmentation is related to axonal pathfinding and the establishment of afferent order (e.g., 16.21-23). Both the Purkinje cell compartments ${ }^{19.20}$ and at least rudimentary mossy fiber compartments ${ }^{25}$ are present in the cerebellar white matter at birth, prior to the onset of granule cell migration, and hence prior to normal synaptogenesis. The natural conclusion is that non-synaptic interactions in the axon tracts are responsible for at least the gross features of axon compartmentation. This is reinforced by the extensive studies of myeloarchitecture ${ }^{26-29}$ showing the presence of alternating, parasagittal axonal compartments in the mature white matter that presumably reflect the migratory histories of the afferent and efferent growth cones.

\section{Afferent Input Does Not Regulate Zebrin I Expression}

Given the close interrelationships between afferent compartments and intrinsic cerebellar cortical markers (above), it is tempting to speculate that ordered afferent input, such as the one from the inferior olive, could take control of the differentiation pathway of an initially homogeneous target field and generate a corresponding pattern of differentiation in the Purkinje cells. This is superficially plausible for three reasons: 1) there is a strong correlation between the positions of the cortical bands in the adult olivocerebellar projection and the antigenic bands revealed by anti-zebrin $I ; ;^{16} 2$ ) the maturation of the climbing fiber projection ${ }^{30-33}$ crudely parallels the development of differential zebrin I expression, and 3) the climbing fiber compartments are present in the white matter prior to synaptic contact with the Purkinje cells..$^{30.34}$ To test this, selected afferent inputs were lesioned and then alterations were sought in the pattern of zebrin I compartmentation. No changes have ever been observed in the adult cerebellar bands as a result of selective 
lesioning in the adult: the number of bands, their relative sizes and their relative positions were unaffected, except where abnormal lobulation made the latter unavoidable. The range of lesions was not exhaustive, but did include bilateral climbing fiber ablation and selective mossy fiber lesions bilaterally and unilaterally. ${ }^{21}$ The same holds for lesions in the afferent cerebellar projections of the newborn rat.

Lesion studies in the neonate seem to preclude a role for afferent inputs in the regulation of zebrin expression postnatally, but a prenatal role in the commitment of Purkinje cells to their mature phenotypes is still possible. To explore this, cerebellar anlagen were dissected from embryos at E12-E15, that is, prior 10 any contact with afferents, and transplanted ectopically into adult hosts, ${ }^{35}$, either into the anterior chamber of the eye, or into cavities prepared in the neocortex. Zebrin I was expressed by grafted Purkinje cells in cortico and in oculo, and doublelabelling experiments confirm that both the zebrin ${ }^{+}$and the zebrin- phenotypes are present. We conclude that afferent input does not play a role in the determination of the zebrin phenotype of Purkinje cells.

If afferent regulation is not the source of Purkinje cell compartmentation, then where does it come from? One possibility is that bands of zebrin $\mathrm{I}^{+}$and zebrin $\mathrm{I}^{-}$Purkinje cells arise by clonal expansion from a small number of committed precursor cells. For example in chimeric mice, a fine-grained mosaicism of non-random clonal compartments has been described in the mature cerebellar cortex. ${ }^{36}$ The relation, if any, between the zebrin I compartmentation and the clonal compartments is still not clear (see also ${ }^{37}$ ). If all Purkinje cells arise from a few early precursors, each already committed to (but not yet expressing) its zebrin I phenotype, then such a ground plan might well be very stable throughout evolution. Early embryonic patterns of development are difficult to change as their ramifications for later development tend to be wide, and hence the consequences of change tend to be fatal. Thus, we would expect the observed similarities in cerebellar ground plan across widely different species. On the other hand, differences in the extent of clonal expansion can be accommodated more easily, and would result in different species having a common set of cerebellar compartments, but of widely differing sizes.

Although compartments are seen in the olivocerebellar projection from birth and thus predate the zebrin compartments, the evidence suggests no dependence of Purkinje compartmentation upon afferent input either in the adult or during postnatal development. Rather, it seems that the maturation of the cerebellum requires the precise matching of topographically organized afferent inputs to pre-defined cerebellar modules. The matching of mossy fiber terminal fields to appropriate Purkinje cell compartments is more complex, as there is no direct synaptic interaction between mossy fiber and Purkinje cell in the adult. It seems likely that the early mossy fiber compartments arise primarily through interactions in the white matter tracts, much as for the olivocerebellar climbing fibers. Once within the target area, the differences in the terminal field distributions might arise either through physiological or molecular mechanisms. For example, the circuitry could be sculpted by competitive interactions. The protracted maturation of the mossy fiber-granule cell circuitry during the first postnatal month, and probably beyond, provides an ideal substrate for experience/activity-dependent refinement of cerebellar circuitry, during which time synapses at inappropriate loci would be eliminated.

\section{Conclusions}

We suggest that far from being a cytologically homogeneous structure, the cerebellar cortex is subdivided sagittally into bands (as defined by using zebrin immunocytochemistry), and further subdivided mediolaterally by the lobulation, and the mossy fiber terminal field distributions. The result is an array of some $10^{3}$ discrete modules, each consisting of around 300 Purkinje cells and their associated afferents and interneurons. Cerebellar afferents are so diverse that each such module could receive a unique pattern of innervation. Furthermore, even if modules received identical inputs they might well terminate differently in the cerebellar and vestibular nuclei, overlapping in different combinations to generate unique composite terminal fields with different patterns of extracerebellar connectivity. The modular segregation of pathways could permit a much higher degree of local structural and functional specialization than could occur in a larger, more homogeneous structure. The total output from the $10^{3}$ modules at any one time - some very active, others quieter - would encode the body global motor state, and through experience, particular patterns of module activity could become correlated with specific motor activities and therefore be used as a reference set for motor learning and control processes.

\section{ACKNOWLEDGEMENTS}

We wish to thank Estrella Gonzalez and Winnie Ho for their assistance, and acknowledge the financial support of the Medical Research Council of Canada and the Alberta Heritage Foundation for Medical Research.

\section{REFERENCES}

1. Shambes GM, Beerman DH, Welker W. Multiple tactile area in cerebellar cortex: another patchy cutaneous projection to granule cell columns in rat. Brain Res 1978; 157: 123-128.

2. Shambes GM, Gibson JM, Welker W. Fractured somatotopy in granule cell tactile areas of rat cerebellar hemispheres revealed by micromapping. Brain Behav Evol 1978; 15:94-140.

3. Hawkes R, Colonnier M, Leclerc N. Monoclonal antibodies reveal sagittal banding in the rodent cerebellar cortex. Brain Res 1985; 333: $359-365$.

4. Hawkes R, Leclerc N. Antigenic map of the rat cerebellar cortex: the distribution of parasagittal bands as revealed by monoclonal anti-Purkinje cell antibody mabQ113. J Comp Neurol 1987; 256: $29-41$

5. Brochu G, Maler L, Hawkes R. Zebrin II: a polypeptide antigen expressed selectively by Purkinje cells reveals compartments in rat and fish cerebellum. J Comp Neurol 1990; 291: 538-552.

6. Plioplys AV, Hawkes R. A survey of mabQ113 immunoreactivity in the adult rat brain: differential staining of the lateral and medial habenular nuclei. Brain Res 1986; 375: 1-12.

7. Doré $\mathrm{L}$, Jacobson $\mathrm{CD}$, Hawkes $\mathrm{R}$. The organization and postnatal development of zebrin II antigenic compartmentation in the cerebellar vermis of the grey opossum, Monodelphis domestica. $\mathbf{J}$ Comp Neurol 1990; 291: 431-449.

8. Lannoo MJ, Brochu G, Maler L, et al. Zebrin Il immunoreactivity in the rat and in the weakly electric teleost Eigenmannia (Gymnotiformes) reveals three modes of Purkinje cell development. J Comp Neurol 1991; 310: 215-233.

9. Plioplys AV, Thibault J, Hawkes R. Selective staining of a subset of Purkinje cells in human cerebellum with monoclonal antibody mabQ113. J Neurol Sci 1985; 70: 245-256.

10. Eisenman LM, Hawkes R. 5'-nucleotidase and the mabQ113 antigen share a common distribution in the cerebellar cortex of the mouse. Neuroscience 1990; 31: 231-235. 
11. Boegman R, Parent A, Hawkes, R Zonation in the rat cerebellar cortex: patches of high acetylcholinesterase activity in the granular layer are congruent with Purkinje cell compartments. Brain Res 1988; 448: 237-251.

12. Leclerc N, Doré L, Parent A, Hawkes R. The compartmentation of the monkey and rat cerebellar cortex: zebrin I and cytochrome oxidase. Brain Res 1990; 506: 70-78.

13. Ingram VI, Ogren MP, Chatot $\mathrm{CL}$, et al. Diversity among Purkinje cells in the monkey cerebellum. Proc Natl Acad Sci U.S.A. 1985; 82: 7131-7135.

14. Leclerc N, Schwarting G, Herrup K, Hawkes R, Yamamoto M. Compartmentation in mammalian cerebellum: zebrin II and $P$ Path antibodies define three classes of sagittally organized bands of Purkinje cells. Proc Natl Acad Sci U.S.A. 1992; 89: 50065010.

15. Eisenman LM. Organization of the olivocerebellar projection to the uvula in the rat. Brain Behav Evol 1984; 24: 1-12.

16. Gravel C, Eisenman LM, Sasseville R, et al. Parasagittal organization of the rat cerebellar cortex: direct correlation between antigenic Purkinje cell bands revealed by mabQ113 and the organization of the olivocerebellar projection. J Comp Neurol 1987; 265: 294-310.

17. Gravel C, Hawkes R. Parasagittal organization of the rat cerebellar cortex: direct comparison of Purkinje cell compartments and the organization of the spinocerebellar projection. J Comp Neurol 1990; 291: 79-102.

18. Hawkes R, Leclerc N. Purkinje cell axon collateral distributions reflect the chemical compartmentation of the rat cerebellar cortex. Brain Res 1989; 476: 279-290.

19. Wassef M, Sotelo C. Asynchrony in the expression of guanosine $3^{\prime}: 5^{\prime}$-phosphate-dependent protein kinase by clusters of Purkinje cells during the perinatal development of rat cerebellum. Neuroscience 1984; 13:1217-1241.

20. Wassef M, Zanetta JP, Brehier A, et al. Transient biochemical compartmentalization of Purkinje cells during early cerebellar development. Dev Biol 1985; 111: 129-137.

21. Leclerc N, Gravel C, Hawkes R. Development of parasagittal zonation in the rat cerebellar cortex: mabQ113 antigenic bands are created postnatally by the suppression of antigen expression in a subset of Purkinje cells. J Comp Neurol 1988; 273: 399-420.

22. Sotelo C. Cerebellar synaptogenesis and the organization of afferent projection maps. Pontificae Acad Scient Scripta Varia 1987, 59: $65-90$.

23. Sotelo C, Bourrat F, Triller A. Postnatal development of the inferior olivary complex in the rat. II. Topographic organization of the immature olivocerebellar projection. J Comp Neurol 1984; 222:177-199.

24. Lannoo MJ, Ross L, Maler L, et al. Development of the cerebellum and its extracerebellar Purkinje cell projection in teleost fishes as determined by zebrin II immunocytochemistry. Prog Neurobiol 1991; 37: 329-363.

25. Arsénio Nunes ML, Sotelo C. Development of the spinocerebellar system in the postnatal rat. J Comp Neurol 1985; 237: 291-306.

26. Voogd J. Comparative aspects of the structure and fibre connections of the mammalian cerebellum. Prog Brain Res 1967; 25: 94-135.

27. Voogd J. The importance of fiber connections in the comparative anatomy of the mammalian cerebellum. In: Llinás $\mathrm{R}$, ed. Neurobiology of Cerebellar Evolution and Development. Chicago: Amer Med Assoc 1969; 493-514.

28. Voogd J, Gerrits NM, Marani E. Cerebellum. In: Paxinos G, ed. The Rat Nervous System. New York: Academic Press 1985; (2) 251-291.

29. Marani E. Topographic enzyme histochemistry of the mammalian cerebellum: 5'-nucleotidase and acetylcholinesterase. Thesis, University of Leiden 1982.

30. Crepel F. Maturation of climbing fiber responses in the rat. Brain Res 1971; 35: 272-276.

31. Crepel F. Regression of functional synapses in the immature mammalian cerebellum. Trends Neurosci 1982; 5: 266-269.

32. Crepel F, Mariani J, Delhaye-Bouchard N. Evidence for a multiple innervation of Purkinje cells by climbing fibers in the immature rat cerebellum. J Neurobiol 1976; 7: 567-578.

33. Mariani J, Changeux J-P. Ontogenesis of olivocerebellar relationships. 1. Studies by intracellular recordings of the multiple innervation of Purkinje cells by climbing fibers in the developing rat cerebellum. J Neurosci 1981; 1: 696-702.

34. Puro DG, Woodward DJ. Maturation of evoked climbing fiber input to rat Purkinje cells. Exp Brain Res 1977; 28: 85-110.

35. Wassef $M$, Sotelo $C$, Thomasset $M$, et al. Expression of compartmentation antigen zebrin I in cerebellar transplants. J Comp Neurol 1990; 294: 223-234.

36. Oster-Granite ML, Gearhart J. Cell lineage analyses of Purkinje cells in murine chimeras. In: Palay SL, Chan-Palay V, eds. The Cerebellum. New Vistas. Berlin, Heidelberg; New York: Springer-Verlag 1982; 75-92.

37. Herrup $K$, Leclerc $N$, Drinkwater $D$, et al. Purkinje cell lineage map is congruent to the antigenic map of zebrin II in the mouse cerebellum. 20th Annual Meeting, Neurosci Soc 1990; 16: 174. 